\title{
Oxygen metabolism and barrier regulation in the intestinal mucosa
}

\author{
Louise E. Glover, J. Scott Lee, and Sean P. Colgan \\ Department of Medicine and the Mucosal Inflammation Program, University of Colorado School of Medicine, Aurora, Colorado, USA.
}

\begin{abstract}
Mucosal surfaces are lined by epithelial cells and provide an important barrier to the flux of antigens from the outside. This barrier is provided at a number of levels, including epithelial junctional complexes, mucus production, and mucosa-derived antimicrobials. Tissue metabolism is central to the maintenance of homeostasis in the mucosa. In the intestine, for example, baseline $\mathrm{pO}_{2}$ levels are uniquely low due to counter-current blood flow and the presence of large numbers of bacteria. As such, hypoxia and HIF signaling predominates normal intestinal metabolism and barrier regulation during both homeostasis and active inflammation. Contributing factors that elicit important adaptive responses within the mucosa include the transcriptional regulation of tight junction proteins, metabolic regulation of barrier components, and changes in autophagic flux. Here, we review recent literature around the topic of hypoxia and barrier function in health and during disease.
\end{abstract}

\section{Introduction}

The mucosa provides a physical barrier between biologic compartments, preventing the free mixing of luminal antigenic material with the lamina propria, which houses the mucosal immune system. The establishment and maintenance of a selectively permeable barrier occurs through interactions of the extracellular domains of multiple transmembrane adhesion proteins between adjacent cells (adherens junction, tight junction, gap junction) or between the mucosal layer and extracellular matrix components (1). These interactions not only determine the physical integrity of the tissue, but also establish the physical organization of lipids and proteins within the plasma membrane in a polarized fashion.

The epithelium overlying all mucosal tissues is supported by a rich vasculature. In these settings, even small perturbations in blood flow can result in relatively large decreases in $\mathrm{O}_{2}$ delivery (hypoxia) to the supporting epithelium. Studies in the mucosa have provided important insight into metabolic demands associated with inflammatory responses. The GI tract, for example, is characterized by a highly unique oxygenation profile, wherein the small and large intestine adapt to significant fluctuations in blood perfusion on a regular basis (2). Even at baseline, epithelial cells lining the mucosa exist at a relatively low $\mathrm{pO}_{2}$, herein described as "physiologic hypoxia" (3). Elucidation of countercurrent $\mathrm{O}_{2}$ exchange mechanisms in the small intestine has revealed that $\mathrm{O}_{2}$ from the arterial blood supply diffuses to adjacent venules along the crypt villus axis, resulting in graded regions of significant hypoxia (4). In the colon, a steep $\mathrm{O}_{2}$ gradient exists from the anaerobic lumen, across the epithelium, and into the vascularized subepithelial mucosa (5). Given the high-energy requirement of the gut and the integral role of the epithelium in maintaining intesti-

Conflict of interest: The authors have declared that no conflict of interest exists. Reference information: J Clin Invest. 2016;126(10):3680-3688. doi:10.1172/JCI84429. nal homeostasis, it is not surprising that these tissues have evolved a number of mechanisms to cope with this austere metabolic environment. Here, we discuss how the intestinal mucosa functionally adapts to the low $\mathrm{pO}_{2}$ environment in health and during disease.

\section{Oxygen utilization in the mucosa}

A comparison of mucosal tissue oxygenation reveals some rather stark contrasts. Fine measurements of the healthy lung alveolus have revealed a surface $\mathrm{pO}_{2}$ of 100 to $110 \mathrm{mmHg}$ (6). By contrast, the most luminal aspect of the healthy colon exists at a $\mathrm{pO}_{2}$ of less than $10 \mathrm{mmHg}(5,7)$. Such differences reflect a combination of $\mathrm{O}_{2}$ sources, the anatomy of blood flow within the organ, and the presence of commensal microbes (8).

Local $\mathrm{pO}_{2}$ environments can be tracked in vivo using 2-nitroimidazole dyes, a class of compounds that undergo intracellular metabolism dependent on levels of cellular $\mathrm{O}_{2}$ content $(9,10)$. Nitroimidazoles form adducts with thiol groups in proteins, peptides, and amino acids. These adducts are retained at $\mathrm{pO}_{2}$ levels of less than $10 \mathrm{mmHg}$ and have the added advantage of imaging only viable tissue (11). Localization of nitroimidazole adducts using antibodies has resulted in two observations (see Figure 1). First, in the normal GI mucosa, particularly in the colon, physiologic hypoxia predominates (7). These studies have shown that these low $\mathrm{pO}_{2}$ conditions are critical for the constitutive expression of some innate immune factors (12) and certain epithelial tight junction proteins (13) (discussed below). Second, inflammatory lesions observed in models of colitis are profoundly hypoxic (or even anoxic), with $\mathrm{pO}_{2}$ levels that are comparable to the $\mathrm{pO}_{2}$ levels observed in some large tumors (7). While there are multiple contributing factors (i.e., increased $\mathrm{O}_{2}$ consumption, vasoconstriction, edema) that result in decreased $\mathrm{O}_{2}$ delivery and resultant hypoxia (7), it was recently shown that a major component of deep tissue hypoxia in active inflammation is derived from NADPH oxidase-dependent $\mathrm{O}_{2}$ consumption by activated leukocytes, particularly neutrophils (14). 
A

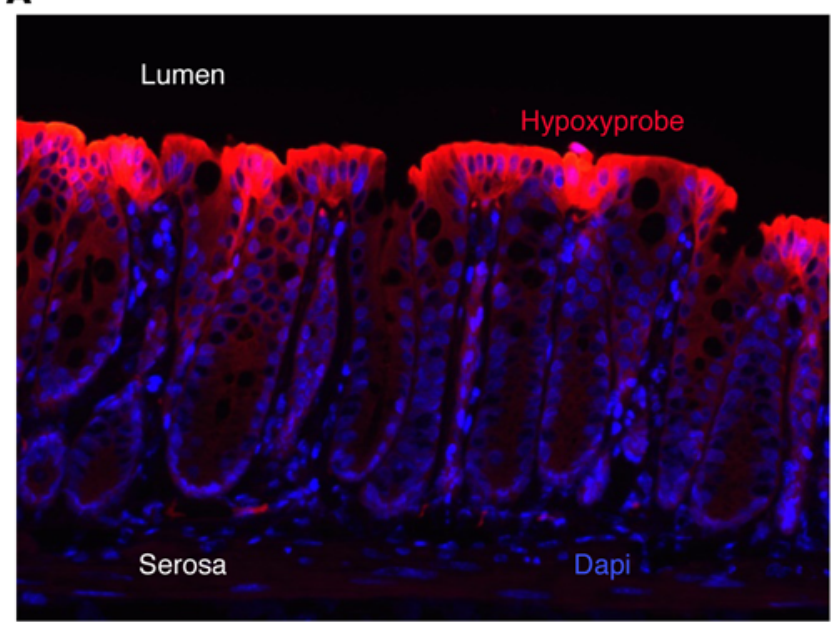

B

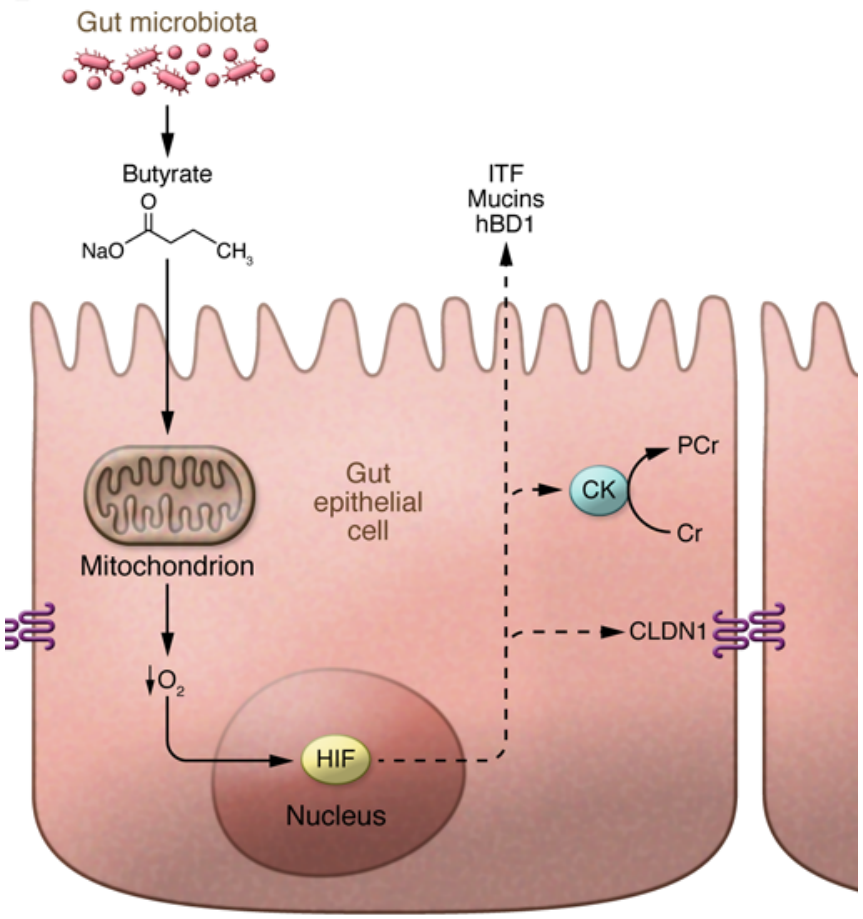

Given the substantial shifts in metabolism and $\mathrm{O}_{2}$ availability during inflammation, a number of studies have shown that stabilization of HIF in low $\mathrm{O}_{2}$ triggers the expression of genes that enable epithelial cells to function as an effective barrier (15). HIF is a member of the Per-ARNT-Sim (PAS) family of basic helix-loop-helix (bHLH) transcription factors and is considered one of the central regulators of tissue metabolism (16). HIF activity is dependent on modifications to the $\mathrm{O}_{2}$-dependent degradation (ODD) domain expressed on the $\alpha$ subunit and subsequent nuclear translocation to form a functional complex with HIF-1 $\beta$ and cofactors such as CREB-binding protein (CBP) and its ortholog p300 (17). Under conditions of normal $\mathrm{O}_{2}$ supply, iron and $\mathrm{O}_{2}$-dependent hydroxylation of two prolines (Pro564 and Pro402) within the ODD of the HIF- $\alpha$ subunit initiates the association with the von Hippel-Lindau tumor suppressor protein $(\mathrm{pVHL})$ and rapid degradation via ubiquitin-E3
Figure 1. Physiologic hypoxia and barrier regulation in the healthy intestinal mucosa. (A) Localization of low $\mathrm{pO}_{2}$ along the crypt-villus axis and the presence of "physiologic hypoxia." Colonic mucosa of healthy mice retain small amounts of nitroimidazole adduct (Hypoxyprobe) that is detected along the luminal aspect of the colon (red). Blue depicts nuclei counterstaining with Dapi. (B) A mechanism in which microbially derived SCFAs, such as butyrate, elicit increased $\mathrm{O}_{2}$ consumption to the extent that HIF is stabilized and transcriptionally active. Also shown here are barrier-related pathways that are directly regulated by HIF. (Cr, creatine; $\mathrm{PCr}$, phosphocreatine; HIF, hypoxia-inducible factor; CK, creatine kinase; CLDN1, claudin-1; ITF, intestinal trefoil factor).

ligase proteasomal targeting (18). To date, three HIF- $\alpha$ subunits have been identified, with the highest level of sequence conservation between HIF-1 $\alpha$ and HIF-2 $\alpha$ (19). Despite their concurrent expression in many cell types, HIF-1 and HIF-2 play nonredundant roles (20) that appear to be highly cell specific to facilitate both short- and long-term adaptations to hypoxia (21). A second HIF activity controller operates in the carboxy terminal transactivation domain of HIF- $\alpha$ subunit, where hypoxia blocks the hydroxylation of Asp80, thereby facilitating the recruitment of $\mathrm{CBP} / \mathrm{p} 300$ (22).

\section{Molecular aspects of barrier regulation in the mucosa}

As previously mentioned, a major function of the mucosa is the provision of a physical barrier between the inside and outside world. This barrier is maintained by cell-cell interactions known as tight junctions (TJs), which serve to seal together the epithelial monolayer (2). TJs are composed of both cytosolic (i.e., zonula occludens) and integral membrane proteins (i.e., occludin, claudins). Adjacent to the $\mathrm{TJ}$ is the adherens junction (AJ), composed of E-cadherin and cadherin/catenin complexes that are linked with the actin cytoskeleton (23). The cytoskeletal links that support apical epithelial junctions are among the most highly ordered arrays of actin filaments in nature (24). The circumferential actomyosin ring mediates selective barrier function in both health and disease (25) and is disrupted by diverse inflammatory stimuli $(26,27)$. In the GI mucosa, such a barrier exists in the presence of trillions of microbes that play a symbiotic role within the host and also serve as a source of disease during dysbiosis (discussed below). Here we discuss the contributions of various components to barrier function and their regulation by the hypoxic microenvironment that has come to characterize the mucosal interface (summarized in Figure 1).

Adherens junctions and hypoxia. Older evidence from both ischemic organs and ATP depletion models described the dissolution of AJ complexes (28), likely initiated by the hyperphosphorylation of the catenins (29). During recovery, epithelia depend on reassembly of the AJ proteins, which necessitates resynthesis of E-cadherin, reassembly of catenins, and reformation of functional AJs. Considering misfolding and/or aggregation of membrane proteins such as E-cadherin in hypoxia and/ or ATP depletion, this could be a limiting factor in the face of sustained hypoxia. Conversely, it has been argued that in some tumors, microenvironmental factors such as hypoxia may result in downregulation of functional surface $\mathrm{AJ}$ complexes and facilitate a metastatic disease phenotype (30). 


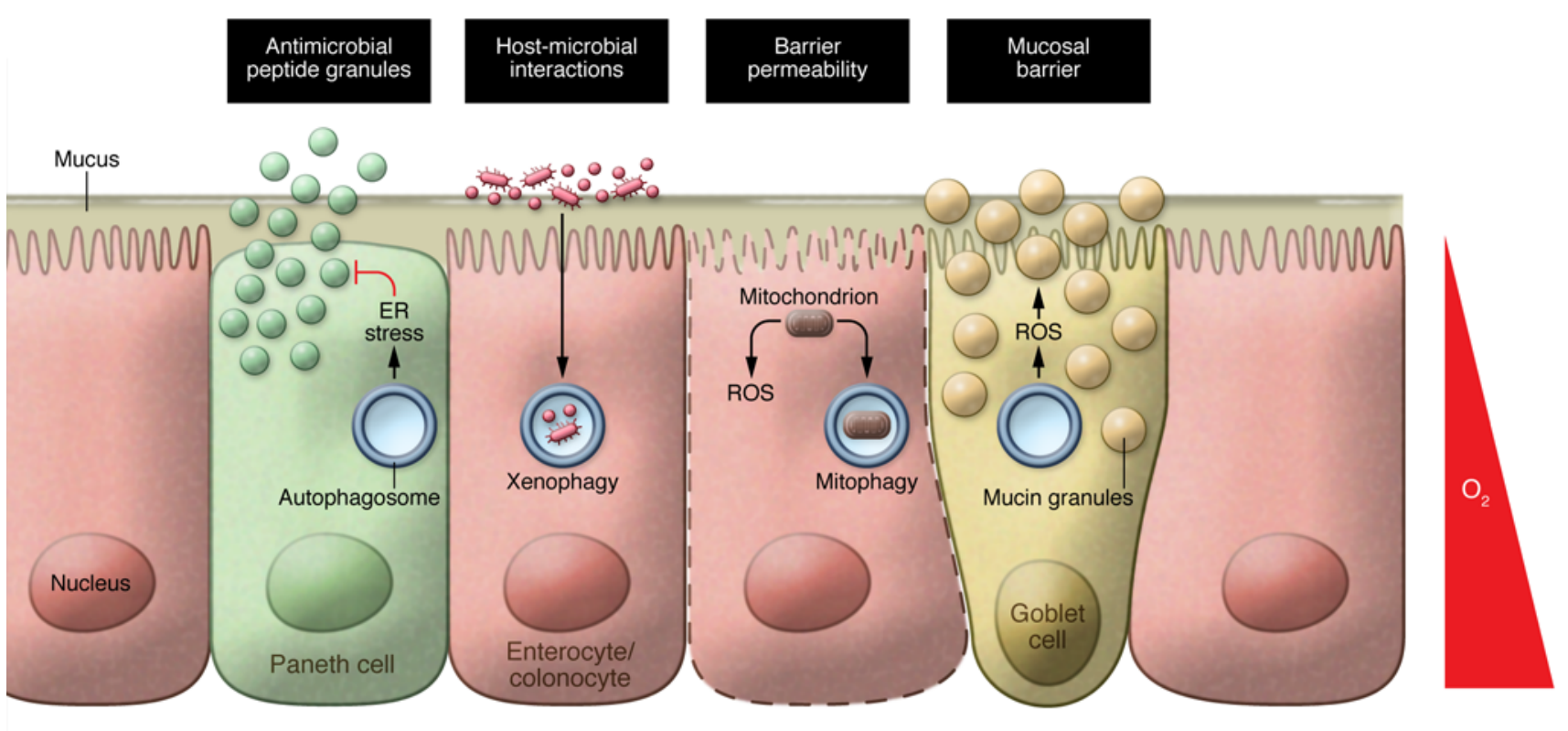

Innate immune regulation

Figure 2. Epithelial autophagy and barrier function. IBD results from dysregulated microbial, epithelial, and immune cell interactions. Autophagy is a key host defense mechanism for epithelial barrier protection against invasive bacteria and metabolic stressors during mucosal inflammation. IBD-associated mutations in autophagy genes have been linked to dysregulated IEC mitophagy, xenophagy, goblet cell mucus secretion, and paneth cell ER stress responses. Although the role of HIF in IEC-intrinsic autophagy in vivo has not yet been characterized, HIF has been identified as a transcriptional regulator of mitochondrial autophagy and xenophagy of IBD-associated AIEC under hypoxic conditions in vitro.

$\beta$-Catenin signaling through T cell factor/lymphoid enhancer factor (TCF/LEF) transcription factors plays a notable role within $\mathrm{AJs}$ and in barrier regulation during active mucosal inflammation (31). Studies derived from microarray analysis of pulmonary epithelial cells following neutrophil (polymorphonuclear leukocyte $[\mathrm{PMN}]$ ) transmigration revealed epithelial gene programming that results in activated $\beta$-catenin signaling and the restoration of epithelial barrier function (31). Such signaling required leukocyte elastase-dependent cleavage of E-cadherin. Parallel studies in the GI mucosa revealed that PMN transmigration elicited a prominent induction of HIF target genes within the epithelium (14). Studies utilizing HIF reporter mice, strategies that deplete circulating $\mathrm{PMN}$, and transgenic mice lacking a respiratory burst (Gp91phox-/), revealed that recruited neutrophils rapidly deplete the local milieu of molecular $\mathrm{O}_{2}$ in an NADPH oxidase-dependent manner. It was shown that transmigrating PMNs "transcriptionally imprint" a molecular signature that reflects PMN-mediated stabilization of HIF and transactivation of HIF targets in the surrounding tissue. This molecular signature promotes an adaptive HIF-dependent resolution of acute inflammation. Indeed, $G p 91^{\text {phox-l- }}$ mice developed more severe colitic responses compared with WT mice, including exaggerated PMN infiltration, diminished inflammatory hypoxia, and increased microbial invasion. Clinical corollaries exist for this model. For example, patients that lack a functional NADPH oxidase (i.e., chronic granulomatous disease [CGD]) often present with an inflammatory bowel disease-like (IBD-like) syndrome (32). Approximately $40 \%$ of CGD patients develop this IBD-like syndrome (33), suggesting that CGD-associated IBD could represent a failure to resolve acute intestinal insults. Like- wise, a number of SNPs that impact the NADPH oxidase complex are IBD candidate genes that are involved in very early onset IBD (34). This NADPH oxidase complex is responsible for the generation of ROS, which are used by innate immune cells (especially PMNs and monocytes/macrophages) as antimicrobial weapons. These studies provide new insight into how cells communicate within tissues and how such information might be harnessed for therapeutic purposes.

Original studies using ATP depletion models revealed an important role for high-energy phosphates in the regulation of barrier function $(35,36)$. These studies have prompted further analysis of hypoxic adaptation to such conditions. Notably, cytosolic creatine kinase (CK) genes are HIF-2-selective targets that are expressed within the AJs of confluent intestinal epithelia, and multiple lines of evidence indicate that CK contributes fundamentally to the energy requirements of barrier regulation (37). The CK pathway is often neglected in energy metabolism, as it is assumed that high-energy phosphate exchange between sites of ATP production (mitochondria/glycolysis) and ATP consumption (ATPases) rely primarily on the diffusion of ATP and ADP. While this may hold true in tissues devoid of CK and phosphocreatine, it is clearly inadequate in tissues with fluctuating energy demands (38). Studies in the mucosa revealed that each of the CK subunits is expressed in cultured intestinal epithelial cell lines, murine colonic epithelia, and in human colonic epithelia (37).During periods of high energy demand, the phosphocreatine pool and CK work to maintain constant concentrations of ATP and ADP. As such, CK functions as a buffer for cytosolic phosphorylation potential that appears to be critical for the proper functioning of a variety of cellular ATPases 
(38). High energy expenditure within the mucosa is particularly relevant during the restoration of epithelial cells following insult (e.g., active inflammation). The dynamic regulation of epithelial junctions is tightly linked to the circumferential F-actin belt (39). From this perspective, it is not surprising that creatine/phosphocreatine may well be central to energy homeostasis and tissue barrier function during episodes of mucosal inflammation.

Tight junctions and hypoxia. Through interactions with the intracellular cytoskeleton, TJs form the backbone for the structural integrity of the epithelial barrier and constitute the physical basis for a permeability barrier to solutes and ions (40). Furthermore, TJs contribute to the polarized phenotype of epithelia by preventing lipid diffusion between apical and basolateral membrane domains, the so-called "fence function" (40). The TJ is composed of both transmembrane and peripheral membrane proteins tightly linked to the actin-based cytoskeleton (39). TJ complex assembly and transcriptional control of its components are tightly regulated by a variety of physiological and pathophysiological stimuli (1). Ischemia and hypoxia dramatically influence TJ integrity, resulting in loss of transepithelial electrical resistance, which has been observed both in ATP depletion models (41) and in vitro hypoxia models (42-44). Some of the permeability changes are attributable to alterations in the distribution of occludin, zonula occludens- 1 (ZO-1), ZO-2, and cingulin $(36,41)$. Furthermore, TJ integrity is influenced by perturbations of the interaction with the actin-based cytoskeleton $(35,45)$ and by the degradation of membrane cytoskeletal proteins such as ankyrin and fodrin in ATP depletion models (46).

Claudins are a large family of tetraspanning integral membrane proteins that are uniquely responsible for the selective permeability of TJs (40). Claudins are categorized as "leaky" or "tight" with regard to their effect on barrier function (47). Claudin-1 (CLDN1) is an important "tight" claudin and has been shown to be dysregulated in a variety of human diseases, including IBD (40). In a recent screen of TJ targets, CLDN1 was identified as a central mediator of aberrant junctional morphology in HIF$1 \beta$-deficient intestinal epithelial cell (IEC) lines (13). Using both loss- and gain-of-function approaches, this work showed that HIF maintains CLDN1 expression through binding hypoxia response element (HRE) sequences in the gene promoter. The reintroduction of CLDN1 into HIF-1 $\beta$-deficient cells restored barrier function and morphologic abnormalities. Moreover, in vivo analysis revealed the importance of HIF-mediated CLDN1 expression during experimental colitis. These results identify a critical link between HIF and specific TJ function, providing important insight into mechanisms of HIF-regulated epithelial homeostasis (13).

Mucus expression and hypoxia. The intestinal epithelium extends its barrier apically through formation of a mucus layer. Mucus is a complex mixture of glycoproteins secreted by goblet cells that allows delivery of nutrients to the epithelium while preventing direct exposure to luminal contents. At least 10 distinct gel-forming and surface mucins are secreted by goblet cells (48). In healthy mucosa, mucus consists of an adherent layer that is devoid of bacteria and a thicker superficial layer that is many times the diameter of the epithelium $(49,50)$. Hypoxia and HIF regulate several components of the mucus layer. MUC3 is a HIF- $1 \alpha$ target whose product, mucin-3, colocalizes with intestinal trefoil factor (ITF), another barrier-protective molecule characterized by robust trefoil domains $(51,52)$. Young and colleagues analyzed the 5 '-flanking sequences of mammalian MUC5AC orthologs and identified evolutionarily conserved regions within domains proximal to the mRNA coding region (53). In particular, SMAD4 and HIF- $1 \alpha$ bind to the promoter, and mutation of their recognition motifs abolishes promoter function (53).

An under-appreciated function of the mucus layer is to provide a reservoir for secreted antimicrobial peptides, such as defensins (54). Defensins are a class of cysteine-rich antimicrobial peptides that possess broad antimicrobial activity $(55,56)$. Human $\beta$ defensin-1 (hBD1) is notable within the intestinal epithelium because it is constitutively secreted, whereas other antimicrobial peptides are induced by inflammatory mediators (8). Constitutive expression of hBD1 depended on basal HIF- $1 \alpha$ signaling in multiple IEC lines and hBD1 expression correlated with other HIF target genes in human tissues (12). Another distinguishing feature of hBD1 is that the full spectrum of its antimicrobial activity is only revealed when its disulfide bonds are reduced (57). Reduction of the hBD1 disulfide bonds is accomplished by thioredoxin, which colocalizes with hBD1 in the colonic mucus; oxidation of hBD1 is prevented by the low $\mathrm{pO}_{2}$ environment of the lumen (58). Thus, hypoxia and HIF appear to provide important regulatory roles for the expression and function of the mucus layer at multiple levels.

Tissue metabolism, hypoxia, and barrier. Mucosal barrier restitution, coupled with the dynamic regulation of the circumferential F-actin belt during barrier maintenance, requires extensive utilization of the energetically costly actin/myosin cytoskeletal structure $(15,39,59,60)$. As alluded to above, ATP and its associated creatine/phosphocreatine buffering system are metabolites of paramount importance, as exemplified by HIF transactivation of creatine transport and creatine kinase enzymes (37). Glutamine is also a fundamental component of mucosal cell metabolism and promotes barrier maintenance by transcriptional regulation of proteins involved in cell proliferation, differentiation, apoptosis, autophagy, protein turnover, antioxidant activity, and immunity (61). Other metabolites have demonstrated roles in barrier restoration and maintenance (62), including arginine and tyrosine through focal adhesion kinase-dependent signaling mechanisms (63-65), polyamines biosynthesized from ornithine $(66,67), n-3$ and n- 6 fatty acids $(68,69)$, vitamin D $(70,71)$, and zinc (72). In indirect support of mucosal homeostasis, cysteine, serine, proline, and especially threonine induce mucin synthesis, and thus aid barrier function by bolstering formation of this first line of defense against microbial invasion $(73,74)$.

Certain metabolites also participate in intestinal mucosal cell barrier maintenance by way of inflammation. Notably, HIF regulates the generation of extracellular adenosine via upregulation of CD39 and CD73 and this increase in adenosine enhances antiinflammatory signaling through binding of the HIF-inducible adenosine A2B receptor (75). Another example is the tryptophan metabolite kynurenate, which exerts antiinflammatory properties by inhibiting xanthine oxidase, resulting in decreased production of ROS during hypoxic stress (76). Likewise, glutamate, methionine, and threonine appear to improve colonic mucosal regeneration after insult (77). These examples provide insight into the integrated responses necessary for homeostatic regulation of tissue barrier function. 


\section{Host-microbial metabolism and tissue barrier}

The gastrointestinal tract is home to trillions of microbes that include bacteria, fungi, and viruses. A finely regulated symbiosis exists within the mucosa. In addition to aiding in digestion, microbial communities produce a number of vitamins and fuel sources in the form of short-chain fatty acids (SCFAs), including butyrate, propionate, and acetate. Butyrate can reach luminal concentrations of $30 \mathrm{mM}$ in the colon and represents a preferred metabolic substrate for colonic epithelial cells, and up to $30 \%$ of energy may be derived from butyrate in the healthy colon (78). SCFAs are efficiently absorbed and metabolized by the epithelium (79). In contrast to other SCFAs, very little butyrate is released into portal circulation (78). Butyrate-derived acetyl-CoA is made available for oxidative phosphorylation and becomes a prominent fuel for colonic epithelial cells (80). It was recently revealed by Kelly et al. that butyrate increases epithelial $\mathrm{O}_{2}$ consumption to the extent that cells sense metabolic hypoxia and HIF is stabilized (81). These same studies revealed that the lack of microbiota (using germ-free or antibiotic-treated mice) resulted in the depletion of butyrate and loss of HIF stabilization. The HIF response was rescued by butyrate supplementation. These observations were recently validated and extended to reveal that the depletion of butyrate-producing species depletes local oxygen levels and allows the expansion of aerobic luminal microbes (82). Given the multiple levels of protection afforded by HIF within the mucosa (2), such findings implicate this butyrate/HIF axis as a host/microbe crosstalk pathway wherein SCFAs promote protective signaling in the distal gut.

A homeostatic role for SCFAs in the distal gut during inflammation is likely important for disease $(78,83)$. For example, recent studies investigating dysbiosis in patients with IBD identified lower levels of colonic butyrate and reduced abundance of butyrate-producing organisms (e.g., certain Faecalibacterium and Roseburia genera) with disease (84-86). The importance of butyrate as the preferred epithelial substrate has been highlighted by the finding that mice with mitochondrial polymorphisms resulting in increased oxidative phosphorylation activity are resistant to colitis (87) and inhibition of $\beta$-oxidation elicits colitis-like symptoms (88). It is notable that administration of exogenous butyrate promotes resistance to experimental colitis $(89,90)$, and several trials have evaluated the efficacy of butyrate in the treatment of human, primarily ulcerative, colitis with mixed results (78). Finally, recent studies have indicated that SCFAs can signal through GPCRs to mediate their biological functions $(91,92)$. In mice, deletion of Gpr41 and Gpr43 mediates protective immunity in inflammatory models $(91,92)$. Treatment of mice with propionate promotes colonic protection during inflammation (92) and the major butyrate receptor GPR109a functions to suppress inflammation-associated carcinogenesis (93).

It is notable that other studies have implicated microbial regulation of iron availability as a mechanism of HIF regulation in the intestine. Pathogenic gram-negative bacteria acquire iron from the host through the secretion of siderophores, which chelate iron for incorporation into normal biological processes. Holden et al. demonstrated that bacterial siderophores such as enterobactin chelate iron to the extent that HIF is stabilized and HIF target genes are induced (94). Likewise, it was shown that siderophores from a number of bacterial genera (e.g., Salmonella, Yersenia, Enterobacter) are capable of stabilizing HIF (95). It was also demonstrated that such HIF stabilization within the intestinal epithelium appears to be an adaptive defense mechanism, since mice lacking epithelial HIF-1 $\alpha$ were more susceptible to Yersinia infection. Such studies provide examples of hypoxia-independent activation of HIF pathways that likely have important biological implications in the GI mucosa.

\section{Autophagy and the regulation of barrier function}

The advent of GWAS has greatly enhanced our understanding of the genetic basis of diseases such as IBD and has highlighted the cell-intrinsic host response to enteric bacteria as a central paradigm of disease pathogenesis (96). Variants in several genes that regulate the autophagy pathway have emerged as significant risk alleles for IBD, including autophagy-related 16-like 1 (ATG16L1) $(97,98)$ and immunity-related GTPase family M $(I R G M)(99,100)$. Moreover, metabolic shifts associated with mucosal inflammation have been shown to directly influence autophagic responses, including ER stress, ROS generation, and hypoxia.

Autophagy is traditionally viewed as a primordial cellular degradation pathway that facilitates cell survival under conditions of nutrient deprivation or metabolic stress (101). Cytoplasmic targets are engulfed by a double-membrane vacuole 0.5 to $1.0 \mu \mathrm{m}$ in diameter termed the autophagosome, which subsequently fuses with lysosomes (autolysosome) for resident hydrolase-mediated digestion. Autophagy also mediates selective recruitment and degradation of cellular targets such as senescent or damaged organelles, protein aggregates, and invading microorganisms, whose large size precludes their elimination by the proteasome. Although a marked effort has been made in recent years to define protein complexes and signaling events underlying acute autophagosome formation, regulation of these processes at the transcriptional level is poorly understood, particularly in the intestine. A number of transcription factors are known to orchestrate altered expression of autophagy genes in response to different microenvironmental stimuli, including transcription factor EB (TFEB) $(102,103)$, zinc finger with KRAB and SCAN domains 3 (ZKSCAN3) (104), AMPK (105), and HIF-1 $(21,106)$.

Perturbations in autophagy disrupt multiple aspects of barrier function (see Figure 2). Recent work has defined a central role for autophagy in colonic goblet cell mucus secretion. Conditional epithelial knockout studies of Atg5, Atg7, and $L c 3 b$ in mice revealed that autophagy is required for efficient mucin granule accumulation and secretion from goblet cells (107). Interestingly, this work implicates NADPH oxidase-derived ROS at the autophagosome-endosome interface as critical mediators of mucus secretion. The NLRP6 inflammasome has also recently been linked to autophagy-dependent mucus secretion, with $N \operatorname{lrp} 6^{-1-}$ mice displaying defective goblet cell autophagy, abrogated mucus granule exocytosis, and high susceptibility to enteric infection (108). Importantly, even partial deficiency of the core autophagy protein ATG5 recapitulates goblet cell and mucus layer defects (108), while mice harboring the prevalent Crohn's disease-associated Atg16l1 mutation T300A were found to display morphological anomalies in epithelial cuff colonic goblet cells (109).

Similarly, defects in IBD-linked autophagy gene expression have been closely connected to disrupted granule exocytosis from Paneth cells (Figure 2). This specialized ileal epithelial lineage 
functions in part to regulate the intestinal microbiota through the secretion of antimicrobial peptides, such as defensins. Hypomorphic expression of Atg16l1 (Atg16l1 ${ }^{\mathrm{HM}}$ ) in mice resulted in profound Paneth cell abnormalities including disorganized and diminished granules, diffuse lysozyme staining, and altered transcriptional profiles leading to increased expression of inflammatory mediators (110). Importantly, similar Paneth cell defects have been reported for knockin transgenic mice and CD patients homozygous for the ATG16L1 T300A risk allele $(109,111)$. Although Atg16l1 ${ }^{\mathrm{HM}}$ mice do not develop spontaneous intestinal inflammation, recent work revealed that epithelial ATG16L1-mediated autophagy is necessary to dampen inositol-requiring enzyme $1 \alpha$-regulated (IRE1 $\alpha$-regulated) NF- $\kappa \mathrm{B}$ activation and attenuate ER stress and cell death in intestinal epithelia deficient in X-box binding protein 1 (Xbp1) and the unfolded protein response (UPR) (112). This seminal study highlights a critical interplay between commensal microbiota, the UPR, and autophagy in Paneth cells, such that dysregulated UPR and increased ER stress may in fact define the initiating threshold for intestinal inflammation in patients harboring certain autophagy gene defects.

The role of absorptive enterocytes as innate immune sensors of bacterial invasion has now been demonstrated in a number of in vivo studies (113-115). Invasive pathogenic and commensal species that penetrate the mucus layer and evade antimicrobial peptides can enter the IEC monolayer and replicate intracellularly. As such, IEC-intrinsic mechanisms that limit bacterial replication and systemic dissemination have been the focus of intense investigation, particularly those involved in xenophagy. Altered expression of xenophagic proteins such as IRGM have been noted in epithelial cells from patients with active IBD (116), and cell culture models of the ATG16L1 variant T300A display defective xenophagy of internalized pathogens $(117,118)$. Conditional deletion of Atg16l1 or Atg5 within the IEC compartment in mice resulted in impaired epithelial autophagy with increased numbers of intracellular Salmonella enterica serovar Typhimurium and increased dissemination to systemic sites $(113,114)$, highlighting an essential role for IEC xenophagy in pathogen clearance. Enterocyte autophagy is likely a specialized response to invasive microbes, as germ-free mice infected with invasive, gram-positive commensal Enterococcus faecalis, but not noninvasive Lactobacillus salivarius, displayed bacterial targeting to autophagosomes (113). The Crohn's diseaseassociated opportunistic pathogen adherent-invasive Escherichia coli (AIEC) is also a target of xenophagy in vitro, and recent work has unveiled an interesting link between HIF-1 expression and AIEC survival in cultured IECs (119). Modulation of IEC metabolism and HIF signaling by inflammatory hypoxia and/or microbial species may therefore define a conserved innate response for host protection through autophagic bacterial capture.

\section{Metabolic regulation of epithelial autophagy}

As the mucosal barrier displays such a uniquely dynamic metabolic and oxygenation profile, an important question is how tonally and microbially induced metabolic fluctuations influence autophagy in IECs. Cellular stressors such as nutrient deprivation, ROS, hypoxia, and ER stress are indeed hallmarks of pathogen invasion, but also reflect the spectrum of microenvironmental fluctuations experienced by IECs.
Although the role of HIF in physiologic and inflammatory IEC-intrinsic autophagy in vivo remains largely uncharacterized, strong evidence exists for integrated influences of the immune repertoire, bacteria, and micronutrients on hypoxic IEC signaling. Hypoxic mucosal macrophages from IBD patients exhibit increased HIF-1 and Wnt1 expression, and coculture models revealed that macrophage-derived Wnt1 can activate mTOR and inhibit autophagy in IECs (120). Mucosa-associated microbiota are likely also prominent modulators of potential hypoxia-elicited autophagic responses (see Figure 2). Both commensal and pathogenic species have been shown to influence mucosal $\mathrm{pO}_{2}$ and/or enterocyte $\mathrm{HIF}$ signaling $(95,121$, 122), and ablation of epithelial HIF-1 increases susceptibility to pathogen infection in a number of studies $(95,121)$. Reciprocally, intestinal oxygenation directly shapes the composition of gut microbial communities, and oxidative changes in intestinal inflammation may underlie the dysbiosis characteristic of IBD (5). The recent characterization of iron recycling by autophagy (ferritinophagy) (123) has additional important implications for HIF signaling and pathogen defense at the mucosal barrier. Finally, mitochondrial autophagy is an important determinant of cell viability under hypoxic stress $(124,125)$. Mitochondrial dysfunction, for example, has been described in IBD case studies (126-128). As outlined above, considerable overlap exists between cellular stimuli for selective autophagy of damaged mitochondria (self) and invading microbes (non-self), and identification of the ubiquitin ligase parkin as a common mediator of mitophagy and xenophagy has led to a paradigm shift in concepts of mammalian autophagy initiation (129).

\section{Therapeutic implications}

Given the breadth of diseases that are potentially influenced by HIF activity (e.g., inflammation, ischemia, edema, fibrosis) there is significant interest in the development of pharmacologic compounds that stabilize HIF and enhance the expression of HIF target genes (130). There is, in fact, a significant unmet need for treatment of diseases where blood flow is compromised and tissues become hypoxic. In the GI mucosa, such diseases include ischemic bowel disease, necrotizing enterocolitis, and IBD. Targeting HIF in these instances holds great promise in preserving tissue function and promoting wound healing and recovery (131).

One pharmacologic approach to achieve HIF stabilization is through the inhibition of HIF prolyl hydroxylases (PHDs) (132, 133). Targeting the catalytic domain of PHDs was originally achieved by screening for molecules that interfere with activation cofactors such as 2-oxoglutarate (e.g., dimethyloxalylglycine [DMOG]) (134). The original studies using PHD inhibition in GI inflammation identified a protective role for pharmacologic HIF stabilization in different models of intestinal inflammation. The use of DMOG for the treatment of colitis proved highly effective in abrogating mucosal inflammation (135). A parallel study revealed that a PHD active site blocker (FG-4497) (134) was associated with the abrogation of mucosal inflammation, most prominently related to enhanced barrier function (refs. 135, 136, and see Figure 1). Additional HIF-1-selective stabilizers, such as AKB-4924, have shown antimicrobial actions through enhanced innate immunity 
and likewise demonstrate significant protection in colitis models $(14,137,138)$. Results from these studies in preclinical models indicate that GI diseases may be one of the more promising applications for PHD inhibitor-based therapies.

\section{Conclusions}

The stark differences in baseline $\mathrm{O}_{2}$ tension between mucosal tissues and the profound increase in energy requirements within inflammatory lesions provide a unique opportunity to understand tissue metabolism in health and disease. Studies in model disease systems have provided new insights leading toward a better understanding of inflammatory responses and mechanisms that promote resolution. Of particular relevance is the shift in tissue oxygenation toward hypoxia, and specifically HIF-target pathways that are strongly associated with tissue barrier function and metabolism that contribute fundamentally to inflammatory resolution. A more precise understanding of the common molecular cues, transcriptional programs, and metabolic milieu that selectively regulate the mucosal barrier will provide substantial insight into the role of IECs as innate immune sensors.

\section{Acknowledgments}

This work was supported by NIH grants DK50189/DK104713/ DK095491/DK103712, and BX002182 from the Veterans Administration and by the Crohn's and Colitis Foundation of America.

Address correspondence to: Sean P. Colgan, University of Colorado School of Medicine, 12700 East 19th Ave. MS B-146, Aurora, Colorado 80045, USA. Phone: 303.724.7235; E-mail: Sean.Colgan@ UCDenver.edu.
1. Koch S, Nusrat A. The life and death of epithelia during inflammation: lessons learned from the gut. Annu Rev Pathol. 2012;7:35-60.

2. Colgan SP, Taylor CT. Hypoxia: an alarm signal during intestinal inflammation. Nat Rev Gastroenterol Hepatol. 2010;7(5):281-287.

3. Karhausen J, Haase VH, Colgan SP. Inflammatory hypoxia: role of hypoxia-inducible factor. Cell Cycle. 2005;4(2):256-258.

4. Shepherd AP. Metabolic control of intestinal oxygenation and blood flow. Fed Proc. 1982;41(6):2084-2089.

5. Albenberg L, et al. Correlation between intraluminal oxygen gradient and radial partitioning of intestinal microbiota. Gastroenterology. 2014;147(5):1055-1063.e8.

6. Schaible B, Schaffer K, Taylor CT. Hypoxia, innate immunity and infection in the lung. Respir Physiol Neurobiol. 2010;174(3):235-243.

7. Karhausen J, Furuta GT, Tomaszewski JE, Johnson RS, Colgan SP, Haase VH. Epithelial hypoxia-inducible factor- 1 is protective in murine experimental colitis. JClin Invest. 2004;114(8):1098-1106.

8. Zheng L, Kelly CJ, Colgan SP. Physiologic hypoxia and oxygen homeostasis in the healthy intestine. A Review in the Theme: Cellular Responses to Hypoxia. Am J Physiol Cell Physiol. 2015;309(6):C350-C360.

9. Cobb LM, Nolan J, Butler SA. Distribution of pimonidazole and RSU 1069 in tumour and normal tissues. Br J Cancer. 1990;62(6):915-918.

10. Laughlin KM, et al. Biodistribution of the nitroimidazole EF5 (2-[2-nitro-1H-imidazol-1-yl]-N(2,2,3,3,3-pentafluoropropyl) acetamide) in mice bearing subcutaneous EMT6 tumors. JPharmacol Exp Ther.1996;277(2):1049-1057.

11. Kizaka-Kondoh S, Konse-Nagasawa H. Significance of nitroimidazole compounds and hypoxia-inducible factor-1 for imaging tumor hypoxia. Cancer Sci. 2009;100(8):1366-1373.

12. Kelly CJ, et al. Fundamental role for HIF-1 $\alpha$ in constitutive expression of human $\beta$ defensin- 1 . Mucosal Immunol. 2013;6(6):1110-1118.

13. Saeedi BJ, et al. HIF-dependent regulation of claudin-1 is central to intestinal epithelial tight junction integrity. Mol Biol Cell. 2015;26(12):2252-2262.
14. Campbell EL, et al. Transmigrating neutrophils shape the mucosal microenvironment through localized oxygen depletion to influence resolution of inflammation. Immunity. 2014;40(1):66-77.

15. Colgan SP, Curtis VF, Lanis JM, Glover LE. Metabolic regulation of intestinal epithelial barrier during inflammation. Tissue Barriers. 2015;3(1-2):e970936.

16. Semenza GL. Regulation of metabolism by hypoxia-inducible factor 1. Cold Spring Harb Symp Quant Biol. 2011;76:347-353.

17. Semenza GL. HIF-1, O(2), and the 3 PHDs: how animal cells signal hypoxia to the nucleus. Cell. 2001;107(1):1-3.

18. Tanimoto K, Makino Y, Pereira T, Poellinger L. Mechanism of regulation of the hypoxia-inducible factor- $1 \alpha$ by the von Hippel-Lindau tumor suppressor protein. EMBO J. 2000;19(16):4298-4309.

19. Wenger RH, Stiehl DP, Camenisch G. Integration of oxygen signaling at the consensus HRE. SciSTKE. 2005;2005(306):re12.

20. Ratcliffe PJ. HIF-1 and HIF-2: working alone or together in hypoxia? J Clin Invest. 2007;117(4):862-865.

21. Majmundar AJ, Wong WJ, Simon MC. Hypoxiainducible factors and the response to hypoxic stress. Mol Cell. 2010;40(2):294-309.

22. Lando D, Peet DJ, Whelan DA, Gorman JJ, Whitelaw ML. Asparagine hydroxylation of the HIF transactivation domain a hypoxic switch. Science. 2002;295(5556):858-861.

23. Bhatt T, Rizvi A, Batta SP, Kataria S, Jamora C. Signaling and mechanical roles of E-cadherin. Cell Commun Adhes. 2013;20(6):189-199.

24. Mooseker MS. Organization, chemistry, and assembly of the cytoskeletal apparatus of the intestinal brush border. Annu Rev Cell Biol. 1985;1:209-241.

25. Madara JL, Pappenheimer JR. Structural basis for physiological regulation of paracellular pathways in intestinal epithelia. J Membr Biol. 1987;100(2):149-164.

26. Koch S, Nusrat A. Dynamic regulation of epithelial cell fate and barrier function by intercellular junctions. Ann N Y Acad Sci. 2009;1165:220-227.

27. Turner JR. Intestinal mucosal barrier function in health and disease. Nat Rev Immunol. 2009;9(11):799-809.
28. Bush KT, Keller SH, Nigam SK. Genesis and reversal of the ishcemic phenotype in epithelial cells. J Clin Invest. 2000;106(5):621-626.

29. Schwartz JH, Shih T, Menza SA, Lieberthal W. ATP depletion increases tyrosine phosphorylation of $\beta$-catenin and plakoglobin in renal tubular cells. J Am Soc Nephrol. 1999;10(11):2297-2305.

30. Giehl K, Menke A. Microenvironmental regulation of E-cadherin-mediated adherens junctions. Front Biosci. 2008;13:3975-3985.

31. Zemans RL, et al. Neutrophil transmigration triggers repair of the lung epithelium via $\beta$-catenin signaling. Proc Natl Acad Sci U S A. 2011;108(38):15990-15995.

32. Huang JS, et al. Chronic granulomatous disease caused by a deficiency in $\mathrm{p} 47$ (phox) mimicking Crohn's disease. Clin Gastroenterol Hepatol. 2004;2(8):690-695.

33. Werlin SL, Chusid MJ, Caya J, Oechler HW. Colitis in chronic granulomatous disease. Gastroenterology. 1982;82(2):328-331.

34. Muise AM, et al. NADPH oxidase complex and IBD candidate gene studies: identification of a rare variant in NCF2 that results in reduced binding to RAC2. Gut. 2012;61(7):1028-1035.

35. Bacallao R, Garfinkel A, Monke S, Zampighi G, Mandel LJ. ATP depletion: a novel method to study junctional properties in epithelial tissues. I. Rearrangement of the actin cytoskeleton. J Cell Sci. 1994;107(pt 12):3301-3313.

36. Tsukamoto T, Nigam SK. Tight junction proteins form large complexes and associate with the cytoskeleton in an ATP depletion model for reversible junction assembly. J Biol Chem. 1997;272(26):16133-16139.

37. Glover LE, et al. Control of creatine metabolism by HIF is an endogenous mechanism of barrier regulation in colitis. Proc Natl Acad Sci US A. 2013;110(49):19820-19825.

38. Wyss M, Kaddurah-Daouk R. Creatine and creatinine metabolism. Physiol Rev. 2000;80(3):1107-1213.

39. Ivanov AI, Parkos CA, Nusrat A. Cytoskeletal regulation of epithelial barrier function during inflammation. Am J Pathol. 2010;177(2):512-524.

40. Capaldo CT, Nusrat A. Claudin switching: physiological plasticity of the tight junction. Semin Cell Dev Biol. 2015;42:22-29. 
41. Tsukamoto T, Nigam SK. Role of tyrosine phosphorylation in the reassembly of occludin and other tight junction proteins. Am J Physiol. 1999;276(5 pt 2):F737-F750.

42. Taylor CT, Dzus AL, Colgan SP. Autocrine regulation of epithelial permeability by hypoxia: role for polarized release of tumor necrosis factor $\alpha$. Gastroenterol.1998;114(4):657-668.

43. Friedman GB, Taylor CT, Parkos CA, Colgan SP. Epithelial permeability induced by neutrophil transmigration is potentiated by hypoxia: role of intracellular cAMP.JCell Physiol. 1998;176(1):76-84.

44. Furuta GT, et al. Hypoxia-inducible factor 1-dependent induction of intestinal trefoil factor protects barrier function during hypoxia. J Exp Med. 2001;193(9):1027-1034.

45. Molitoris BA, Leiser J, Wagner MC. Role of the actin cytoskeleton in ischemia-induced cell injury and repair. Pediatr Nephrol. 1997;11(6):761-767.

46. Molitoris BA, Dahl R, Hosford M. Cellular ATP depletion induces disruption of the spectrin cytoskeletal network. Am J Physiol. 1996; 271(4 pt 2):F790-F798.

47. Anderson JM, Van Itallie CM, Fanning AS. Setting up a selective barrier at the apical junction complex. Curr Opin Cell Biol. 2004;16(2):140-145.

48. Linden SK, Sutton P, Karlsson NG, Korolik V, McGuckin MA. Mucins in the mucosal barrier to infection. Mucosal Immunol. 2008;1(3):183-197.

49. Atuma C, Strugala V, Allen A, Holm L. The adherent gastrointestinal mucus gel layer: thickness and physical state in vivo. Am J Physiol Gastrointest Liver Physiol. 2001;280(5):G922-G929.

50. Strugala V, Allen A, Dettmar PW, Pearson JP. Colonic mucin: methods of measuring mucus thickness. Proc Nutr Soc. 2003;62(1):237-243.

51. Louis NA, Hamilton KE, Canny G, Shekels LL, Ho SB, Colgan SP. Selective induction of mucin-3 by hypoxia in intestinal epithelia. JCell Biochem. 2006;99(6):1616-1627.

52. Longman RJ, et al. Coordinated localisation of mucins and trefoil peptides in the ulcer associated cell lineage and the gastrointestinal mucosa. Gut. 2000;47(6):792-800.

53. Young HW, et al. Central role of Muc5ac expression in mucous metaplasia and its regulation by conserved 5' elements. Am J Respir Cell Mol Biol. 2007;37(3):273-290.

54. Antoni L, et al. Human colonic mucus is a reservoir for antimicrobial peptides. JCrohns Colitis. 2013;7(12):e652-e664.

55. Ganz T. Defensins: antimicrobial peptides of innate immunity. Nat Rev Immunol. 2003;3(9):710-720.

56. Pazgier M, Hoover DM, Yang D, Lu W, Lubkowski J. Human beta-defensins. Cell Mol Life Sci. 2006;63(11):1294-1313.

57. Schroeder BO, et al. Reduction of disulphide bonds unmasks potent antimicrobial activity of human $\beta$-defensin 1 . Nature. 2011;469(7330):419-423.

58 . Jaeger SU, et al. Cell-mediated reduction of human $\beta$-defensin 1: a major role for mucosal thioredoxin. Mucosal Immunol. 2013;6(6):1179-1190.

59. Culic O, Gruwel ML, Schrader J. Energy turnover of vascular endothelial cells. Am J Physiol. 1997;273(1 pt 1):C205-C213.

60. Daniel JL, Molish IR, Robkin L, Holmsen H.
Nucleotide exchange between cytosolic ATP and F-actin-bound ADP may be a major energyutilizing process in unstimulated platelets. Eur J Biochem. 1986;156(3):677-684.

61. Wang B, et al. Glutamine and intestinal barrier function. Amino Acids. 2014;47(10):2143-2154.

62. Lan A, et al. Mucosal healing in inflammatory bowel diseases: is there a place for nutritional supplementation? Inflamm Bowel Dis. 2015;21(1):198-207.

63. Guan JL. Role of focal adhesion kinase in integrin signaling. Int J Biochem Cell Biol. 1997;29(8-9):1085-1096.

64. Gookin JL, Rhoads JM, Argenzio RA. Inducible nitric oxide synthase mediates early epithelial repair of porcine ileum. Am J Physiol Gastrointest Liver Physiol. 2002;283(1):G157-G168.

65. Rhoads JM, et al. Arginine stimulates intestinal cell migration through a focal adhesion kinase dependent mechanism. Gut. 2004;53(4):514-522.

66. McCormack SA, Viar MJ, Johnson LR. Polyamines are necessary for cell migration by a small intestinal crypt cell line. Am J Physiol. 1993; 264(2 pt 1):G367-G374.

67. Pegg AE. Mammalian polyamine metabolism and function. IUBMB Life. 2009;61(9):880-894.

68. Ruthig DJ, Meckling-Gill KA. Both (n-3) and (n-6) fatty acids stimulate wound healing in the rat intestinal epithelial cell line, IEC-6. J Nutr. 1999;129(10):1791-1798.

69. Jacobi SK, Moeser AJ, Corl BA, Harrell RJ, Blikslager AT, Odle J. Dietary long-chain PUFA enhance acute repair of ischemia-injured intestine of suckling pigs. J Nutr. 2012;142(7):1266-1271.

70. Kong J, et al. Novel role of the vitamin D receptor in maintaining the integrity of the intestinal mucosal barrier. Am J Physiol Gastrointest Liver Physiol. 2008;294(1):G208-G216.

71. Jørgensen SP, et al. Clinical trial: vitamin D3 treatment in Crohn's disease - a randomized double-blind placebo-controlled study. Aliment Pharmacol Ther. 2010;32(3):377-383.

72. Cario E, et al. Effects of exogenous zinc supplementation on intestinal epithelial repair in vitro. Eur J Clin Invest. 2000;30(5):419-428.

73. Faure M, Moënnoz D, Montigon F, Mettraux C, Breuillé D, Ballèvre O. Dietary threonine restriction specifically reduces intestinal mucin synthesis in rats. J Nutr. 2005;135(3):486-491.

74. Faure M, et al. Specific amino acids increase mucin synthesis and microbiota in dextran sulfate sodium-treated rats. J Nutr. 2006; 136(6):1558-1564

75. Colgan SP, Eltzschig HK. Adenosine and hypoxia-inducible factor signaling in intestinal injury and recovery. Annu Rev Physiol. 2012;74:153-175.

76. Kaszaki J, et al. Kynurenic acid inhibits intestinal hypermotility and xanthine oxidase activity during experimental colon obstruction in dogs. Neurogastroenterol Motil. 2008;20(1):53-62.

77. Liu X, et al. Beneficial effects of an amino acid mixture on colonic mucosal healing in rats. Inflamm Bowel Dis. 2013;19(13):2895-2905.

78. Hamer HM, Jonkers D, Venema K, Vanhoutvin S, Troost FJ, Brummer RJ. Review article: the role of butyrate on colonic function. Aliment Pharmacol Ther. 2008;27(2):104-119.

79. Tan J, McKenzie C, Potamitis M, Thorburn AN,
Mackay CR, Macia L. The role of short-chain fatty acids in health and disease. Adv Immunol. 2014;121:91-119.

80. Blouin JM, et al. Butyrate elicits a metabolic switch in human colon cancer cells by targeting the pyruvate dehydrogenase complex. Int J Cancer. 2011;128(11):2591-2601.

81. Kelly CJ, et al. Crosstalk between microbiotaderived short-chain fatty acids and intestinal epithelial HIF augments tissue barrier function. Cell Host Microbe. 2015;17(5):662-671.

82. Rivera-Chávez F, et al. Depletion of butyrate-producing clostridia from the gut microbiota drives an aerobic luminal expansion of Salmonella. Cell Host Microbe. 2016;19(4):443-454.

83. Plöger S, et al. Microbial butyrate and its role for barrier function in the gastrointestinal tract. Ann N Y Acad Sci. 2012;1258:52-59.

84. Machiels K, et al. A decrease of the butyrate-producing species Roseburia hominis and Faecalibacterium prausnitzii defines dysbiosis in patients with ulcerative colitis. Gut. 2014;63(8):1275-1283.

85. Eeckhaut V, et al. Butyricicoccus pullicaecorum in inflammatory bowel disease. Gut. 2013;62(12):1745-1752

86. Sokol H, et al. Low counts of Faecalibacterium prausnitzii in colitis microbiota. Inflamm Bowel Dis. 2009;15(8):1183-1189.

87. Bär F, et al. Mitochondrial gene polymorphisms that protect mice from colitis. Gastroenterology. 2013;145(5):1055-1063.e3.

88. Roediger WE, Nance S. Metabolic induction of experimental ulcerative colitis by inhibition of fatty acid oxidation. Br J Exp Pathol. 1986;67(6):773-782.

89. Cresci G, Nagy LE, Ganapathy V. Lactobacillus GG and tributyrin supplementation reduce antibiotic-induced intestinal injury. JPEN J Parenter Enteral Nutr. 2013;37(6):763-774.

90. Leonel AJ, et al. Antioxidative and immunomodulatory effects of tributyrin supplementation on experimental colitis. Br J Nutr. 2013;109(8):1396-1407.

91. Kim MH, Kang SG, Park JH, Yanagisawa M, Kim $\mathrm{CH}$. Short-chain fatty acids activate GPR41 and GPR43 on intestinal epithelial cells to promote inflammatory responses in mice. Gastroenterology. 2013;145(2):396-406.e1.

92. Maslowski KM, et al. Regulation of inflammatory responses by gut microbiota and chemoattractant receptor GPR43. Nature. 2009;461(7268):1282-1286.

93. Singh N, et al. Activation of Gpr109a, receptor for niacin and the commensal metabolite butyrate, suppresses colonic inflammation and carcinogenesis. Immunity. 2014;40(1):128-139.

94. Holden VI, Lenio S, Kuick R, Ramakrishnan SK, Shah YM, Bachman MA. Bacterial siderophores that evade or overwhelm lipocalin 2 induce hypoxia inducible factor 1alpha and proinflammatory cytokine secretion in cultured respiratory epithelial cells. Infect Immun. 2014;82(9):3826-3836.

95. Hartmann H, et al. Hypoxia-independent activation of HIF-1 by enterobacteriaceae and their siderophores. Gastroenterology. 2008;134(3):756-767.

96. Jostins L, et al. Host-microbe interactions have shaped the genetic architecture of inflammatory 
bowel disease. Nature. 2012;491(7422):119-124.

97. Hampe J, et al. A genome-wide association scan of nonsynonymous SNPs identifies a susceptibility variant for Crohn disease in ATG16L1. Nat Genet. 2007;39(2):207-211.

98. Rioux JD, et al. Genome-wide association study identifies new susceptibility loci for Crohn disease and implicates autophagy in disease pathogenesis. Nat Genet. 2007;39(5):596-604.

99. McCarroll SA, et al. Deletion polymorphism upstream of IRGM associated with altered IRGM expression and Crohn's disease. Nat Genet. 2008;40(9):1107-1112.

100.Parkes M, et al. Sequence variants in the autophagy gene IRGM and multiple other replicating loci contribute to Crohn's disease susceptibility. Nat Genet. 2007;39(7):830-832.

101.Levine B, Mizushima N, Virgin HW. Autophagy in immunity and inflammation. Nature. 2011;469(7330):323-335.

102.Settembre C, et al. TFEB links autophagy to lysosomal biogenesis. Science. 2011;332(6036):1429-1433.

103. Visvikis O, et al. Innate host defense requires TFEB-mediated transcription of cytoprotective and antimicrobial genes. Immunity. 2014;40(6):896-909.

104. Chauhan S, et al. ZKSCAN3 is a master transcriptional repressor of autophagy. Mol Cell. 2013;50(1):16-28.

105. Liang J, et al. The energy sensing LKB1-AMPK pathway regulates p27(kip1) phosphorylation mediating the decision to enter autophagy or apoptosis. Nat Cell Biol. 2007;9(2):218-224.

106. Bellot G, et al. Hypoxia-induced autophagy is mediated through hypoxia-inducible factor induction of BNIP3 and BNIP3L via their BH3 domains. Mol Cell Biol. 2009;29(10):2570-2581.

107. Patel KK, et al. Autophagy proteins control goblet cell function by potentiating reactive oxygen species production. EMBO J. 2013;32(24):3130-3144.

108. Wlodarska M, et al. NLRP6 inflammasome orchestrates the colonic host-microbial interface by regulating goblet cell mucus secretion. Cell. 2014;156(5):1045-1059.

109.Lassen KG, et al. Atg16L1 T300A variant decreases selective autophagy resulting in altered cytokine signaling and decreased antibacterial defense. Proc Natl Acad Sci US A. 2014;111(21):7741-7746.

110. Cadwell K, et al. A key role for autophagy and the autophagy gene Atg16l1 in mouse and human intestinal Paneth cells. Nature. 2008;456(7219):259-263.

111. Murthy A, et al. A Crohn's disease variant in
Atg16l1 enhances its degradation by caspase 3 . Nature. 2014;506(7489):456-462.

112. Adolph TE, et al. Paneth cells as a site of origin for intestinal inflammation. Nature. 2013;503(7475):272-276.

113. Benjamin JL, Sumpter R Jr, Levine B, Hooper LV. Intestinal epithelial autophagy is essential for host defense against invasive bacteria. Cell Host Microbe. 2013;13(6):723-734.

114. Conway KL, et al. Atg16l1 is required for autophagy in intestinal epithelial cells and protection of mice from Salmonella infection. Gastroenterology. 2013;145(6):1347-1357.

115. Knodler LA, et al. Noncanonical inflammasome activation of caspase-4/caspase-11 mediates epithelial defenses against enteric bacterial pathogens. Cell Host Microbe. 2014;16(2):249-256.

116 . Brest $P$, et al. A synonymous variant in IRGM alters a binding site for miR-196 and causes deregulation of IRGM-dependent xenophagy in Crohn's disease. Nat Genet. 2011;43(3):242-245.

117. Kuballa P, Huett A, Rioux JD, Daly MJ, Xavier RJ. Impaired autophagy of an intracellular pathogen induced by a Crohn's disease associated ATG16L1 variant. PLoS One. 2008;3(10):e3391.

118. Lapaquette P, Glasser AL, Huett A, Xavier RJ, Darfeuille-Michaud A. Crohn's disease-associated adherent-invasive E. coli are selectively favoured by impaired autophagy to replicate intracellularly. Cell Microbiol. 2010;12(1):99-113.

119. Mimouna S, et al. HIF1A regulates xenophagic degradation of adherent and invasive Escherichia coli (AIEC). Autophagy. 2014;10(12):2333-2345.

120. Ortiz-Masiá D, et al. Hypoxic macrophages impair autophagy in epithelial cells through Wnt1: relevance in IBD. Mucosal Immunol. 2014;7(4):929-938.

121. Hirota SA, et al. Hypoxia-inducible factor signaling provides protection in Clostridium difficile-induced intestinal injury. Gastroenterology. 2010;139(1):259-69.e3.

122. Jennewein J, et al. Low-oxygen tensions found in Salmonella-infected gut tissue boost Salmonella replication in macrophages by impairing antimicrobial activity and augmenting Salmonella virulence. Cell Microbiol. 2015;17(12):1833-1847.

123. Mancias JD, Wang X, Gygi SP, Harper JW, Kimmelman AC. Quantitative proteomics identifies NCOA4 as the cargo receptor mediating ferritinophagy. Nature. 2014;509(7498):105-109.

124. Fukuda R, Zhang H, Kim JW, Shimoda L, Dang CV, Semenza GL. HIF-1 regulates cytochrome oxidase subunits to optimize efficiency of respiration in hypoxic cells. Cell. 2007;129(1):111-122.
125. Zhang $\mathrm{H}$, et al. Mitochondrial autophagy is an HIF-1-dependent adaptive metabolic response to hypoxia. J Biol Chem. 2008;283(16):10892-10903.

126. Ostuni MA, et al. Overexpression of translocator protein in inflammatory bowel disease: potential diagnostic and treatment value. Inflamm Bowel Dis. 2010;16(9):1476-1487.

127. Restivo NL, Srivastava MD, Schafer IA, Hoppel CL. Mitochondrial dysfunction in a patient with crohn disease: possible role in pathogenesis. J Pediatr Gastroenterol Nutr. 2004;38(5):534-538.

128.Santhanam S, et al. Mitochondrial electron transport chain complex dysfunction in the colonic mucosa in ulcerative colitis. Inflamm Bowel Dis. 2012;18(11):2158-2168.

129. Manzanillo PS, et al. The ubiquitin ligase parkin mediates resistance to intracellular pathogens. Nature. 2013;501(7468):512-516.

130. Eltzschig HK, Bratton DL, Colgan SP. Targeting hypoxia signalling for the treatment of ischaemic and inflammatory diseases. Nat Rev Drug Discov. 2014;13(11):852-869.

131. Semenza GL. Oxygen sensing, hypoxia-inducible factors, and disease pathophysiology. Annu Rev Pathol. 2014;9:47-71.

132. Epstein AC, et al. C. elegans EGL-9 and mammalian homologs define a family of dioxygenases that regulate HIF by prolyl hydroxylation. Cell. 2001;107(1):43-54.

133. Jaakkola P, et al. Targeting of HIF- $\alpha$ to the von Hippel-Lindau ubiquitylation complex by $\mathrm{O}_{2}$-regulated prolyl hydroxylation. Science. 2001;292(5516):468-472.

134. Fraisl P, Aragonés J, Carmeliet P. Inhibition of oxygen sensors as a therapeutic strategy for ischaemic and inflammatory disease. Nat Rev Drug Discov. 2009;8(2):139-152.

135. Cummins EP, et al. The hydroxylase inhibitor dimethyloxalylglycine is protective in a murine model of colitis. Gastroenterology. 2008;134(1):156-165

136. Robinson A, Keely S, Karhausen J, Gerich ME, Furuta GT, Colgan SP. Mucosal protection by hypoxia-inducible factor prolyl hydroxylase inhibition. Gastroenterology. 2008;134(1):145-155.

137. Keely S, et al. Contribution of epithelial innate immunity to systemic protection afforded by prolyl hydroxylase inhibition in murine colitis. Mucosal Immunol. 2014;7(1):114-123.

138. Okumura CY, et al. A new pharmacological agent (AKB-4924) stabilizes hypoxia inducible factor-1 (HIF-1) and increases skin innate defenses against bacterial infection. JMol Med. 2012;90(9):1079-1089. 